\title{
Low-dose radiation-induced epithelial-mesenchymal transition through NF-kB in cervical cancer cells
}

\author{
SHI YAN ${ }^{1}$, YU WANG $^{1}$, QIFENG YANG ${ }^{2}$, XIAOYAN LI $^{2}$, XIAOLI KONG ${ }^{2}$, \\ NING ZHANG ${ }^{2}$, CUNZHONG YUAN $^{1}$, NING YANG ${ }^{1}$ and BEIHUA KONG ${ }^{1}$ \\ Departments of ${ }^{1}$ Obstetrics and Gynaecology and ${ }^{2}$ Breast Surgery, \\ Qilu Hospital of Shandong University, Jinan, Shandong, P.R. China
}

Received December 20, 2012; Accepted February 18, 2013

DOI: $10.3892 /$ ijo.2013.1852

\begin{abstract}
Cervical cancer is the leading cause of death from cancer among women. Radiotherapy for cervical cancer is an effective treatment method; however, the response to radiotherapy varies among patients. Epithelial-mesenchymal transition (EMT) is a morphogenesis process involved in embryonic and organismal development. During tumour progression, EMT may enhance cancer cell invasion, promoting tumour metastasis. We hy pothesised that EMT was involved in the enhanced invasiveness of cervical cancer cells after low-dose radiation and aimed to elucidate the underlying mechanism of this process in low-dose radiation of cervical cancer. The irradiated cells (FIR cells) were derived from the parental cells ( $\mathrm{N}$ cells) with a cumulative dose of $75 \mathrm{~Gy}$. After resting and reorganisation, the effect of low-dose radiation on the FIR cells was analysed. The expression of E-cadherin, $\mathrm{N}$-cadherin and p65 was detected by real-time qPCR and western blotting in parental cancer cells and irradiated cancer cells. Motility was detected using the migration/invasion assay. After silencing of NF- $\mathrm{kB}$ p65 expression using siRNA against $\mathrm{p} 65$, the expression of $\mathrm{E}$-cadherin and $\mathrm{N}$-cadherin was examined by real-time qPCR and western blotting. We found that low-dose radiation induced morphological changes of cells. The expression of epithelial markers was downregulated and mesenchymal markers were induced in irradiated cells, both of which are characteristics of EMT. Additionally, in irradiated cells, migration and invasion were enhanced and the expression of p65 was increased. To investigate whether p65 was involved in EMT, we silenced the expression of p65 in irradiated cells using siRNA and found that the features of EMT were suppressed. In summary, p65-regulated EMT induced by low-dose irradiation of cervical cancer cell lines
\end{abstract}

Correspondence to: Professor Beihua Kong, Department of Obstetrics and Gynaecology, Qilu Hospital of Shandong University, 107 Wenhua Xi Road, Jinan, Shandong, P.R. China

E-mail: kongbeihua@sdu.edu.cn

Key words: cervical cancer, radiation resistance, low-dose radiation, epithelial-mesenchymal transition promoted the invasiveness and metastasis of cervical cancer cells. The reversal of EMT may be a new therapeutic target for improving the effectiveness of radiotherapy for cervical cancer.

\section{Introduction}

Cervical cancer is the second most prevalent female carcinoma worldwide after breast cancer (1). In 2012, the estimated number of new cases of uterine cervix cancer was 12,170 , accounting for $13.7 \%$ of all gynaecological cancers in the United States (2). Although many therapy methods are available, such as surgery therapy, radiotherapy, chemotherapy and biotherapy and with much improvement in the application of these techniques, death from cervical cancer still accounts for $14.3 \%$ of all gynaecological cancers in the United States. According to the reported deaths for the five leading cancers (breast cancer, uterine cervix cancer, colorectal cancer, leukaemia and brain and other nervous system cancers) by age and gender in the United States in 2008, cervical cancer was the second leading cause of cancer death among women aged 20-39 years, ranked second only to breast cancer (2).

Radiotherapy is an effective therapy method for cervical cancer, particularly when the disease is at an advanced stage. Patients show different responses to this therapy method: some are cured, whereas others suffer failed therapy (3) and are prone to relapse and distant metastasis due to radioresistance. Many factors could impact tumour cells responding to radiotherapy in the microenvironment. Ionising radiation is able to activate sets of genes associated with apoptosis, DNA damage repair, cell adhesion and angiogenesis signalling pathways; these genes, in turn, may mediate cellular responses to radiation $(4,5)$, influencing the effects of radiotherapy. p73 elicited apoptosis in response to DNA damage induced by radiation and it demonstrated significantly higher expression in radiosensitive cancers than in radioresistant cancers. Additionally, p73 might be important in regulating the radiation response in cervical cancer cells (6). Ionising radiation was able to activate transforming growth factor $\beta 1$ (TGF $\beta$ ); in turn, TGF $\beta$ mediates the cellular and tissue radiation response $(7,8)$.

Epithelial-mesenchymal transition (EMT) is a morphogenesis process involved in embryonic and organ development. This process is also involved in carcinoma progression (1). 
The features of EMT include loss of cell adhesion molecules, downregulation of the expression of epithelial differentiation markers and increased expression of mesenchymal markers $(9,10)$. Many factors control this process, including several soluble factors, ion transport systems, cytoskeletal modulators and transcriptional factors. Among these signals, transcription factors Snail, slug and twist play an important role (1) in inducing EMT by repressing E-cadherin transcription (11). Data have shown that the recurrence of breast cancer after therapy is accompanied by EMT (12). Tumourigenic EMT signalling pathways that facilitate the migration and invasion of epithelial cancer cells also contribute to apoptosis resistance and chemoresistant malignant behaviour $(1,13)$. Increasing evidence has suggested that EMT contributes to chemotherapy resistance in cancer cells (14-17). We proposed that EMT plays a role in the low-dose radiation of cervical cancer cells, leading to invasion and metastasis. We also tried to clarify the underlying mechanism that was implicated in this process and we have identified a new strategy to make therapy more specific.

\section{Materials and methods}

Cell lines and reagents. The human cervical cancer cell lines Siha and C33A and mouse fibroblast cell line NIH3T3 were obtained from the American Type Culture Collection (ATCC, Rockville, MD, USA). Dulbecco's modified Eagle's medium (DMEM), minimum essential medium (MEM) and fetal bovine serum (FBS) were purchased from Gibco-BRL (Rockville, IN, USA). Rabbit anti-E-cadherin, anti- $\beta$-catenin, anti-vimentin,

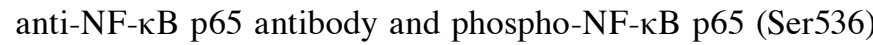
$(93 \mathrm{H} 1)$ rabbit $\mathrm{mAb}$ were purchased from Cell Signaling Technology (Beverly, MA, USA). Mouse anti- $\beta$-actin antibody was purchased from Sigma-Aldrich (St. Louis, MO, USA). TRIzol reagent was purchased from Invitrogen (Carlsbad, CA, USA). Horseradish peroxidase (HRP)-labelled goat antirabbit $\mathrm{IgG}(\mathrm{H}+\mathrm{L})$ antibody and HRP-labelled goat anti-mouse $\operatorname{IgG}(\mathrm{H}+\mathrm{L})$ antibody were obtained from Kirkegaard \& Perry Laboratories, Inc. (KPL, Gaithersburg, MD, USA). Cell lysis buffer for western blotting was purchased from Biocolor BioScience \& Technology Co. (Shanghai, China).

Cell culture and treatment. The human cervical cancer cell lines Siha and C33A were cultured in MEM supplemented with 10\% FBS and the mouse fibroblast cell line NIH3T3 was cultured in DMEM supplemented with $10 \%$ FBS and $100 \mathrm{U}$ of penicillin-streptomycin at $37^{\circ} \mathrm{C}$ in a humidified $5 \% \mathrm{CO}_{2}$ incubator.

Radiation treatment. The cervical cancer cells Siha and C33A cells were cultured in $25-\mathrm{cm}^{2}$ flasks. When the cells reached $75 \%$ confluence, they were treated using the Faxitron Cabinet X-ray System (Faxitron, Wheeling, IL, USA) as previously described (18). The irradiated cells were derived after irradiation of the parental cells at a dose rate of $0.36 \mathrm{~Gy} / \mathrm{min}$ (given in 2-Gy fractions five times/week) with a cumulative dose of 75 Gy. The control cells were treated using the same procedure except that they were unirradiated. The culture medium of the parental cells and X-ray-irradiated cells was changed every 3 days and the cells were passaged every 7 days. After reaching $75 \mathrm{~Gy}$, the surviving cells were allowed to rest and reorganise for 2 weeks before experiments.

RNA extraction and real-time qPCR analysis. Total RNA was isolated using TRIzol reagent according to the manufacturer's protocol and reverse transcriptase reactions were performed using $1 \mu \mathrm{g}$ total RNA and the PrimerScript RT reagent kit (Takara Biotechnology Co., Ltd., Dalian, China). For real-time PCR, the Applied Biosystems StepOnePlus ${ }^{\text {TM }}$ Real-Time PCR System was used according to the manufacturer's protocol. The relative gene expression $\Delta \mathrm{Ct}$ values of mRNA were calculated by normalising to expression of the endogenous control GAPDH. Each experiment was repeated three times.

Cell lysis and western blot analysis. The cells were washed three times with PBS, scraped and lysed in RIPA buffer (Shennengbocai, Shanghai, China) (0.1\% SDS, 5 mM EDTA, $1 \%$ NP-40, $0.5 \%$ sodium deoxycholate and $1 \mathrm{mM}$ sodium vanadate) containing protease inhibitors (1 mM sodium fluoride, $1 \mathrm{mM}$ PMSF). The lysates were harvested on ice for $30 \mathrm{~min}$ and centrifuged at $12,000 \mathrm{rpm}$ for $15 \mathrm{~min}$ at $4^{\circ} \mathrm{C}$. The supernatants were collected and the protein content was measured using the BCA Protein Assay kit (Merck, Darmstadt, Germany). Seventy micrograms of total protein was separated by $5-10 \%$ SDS-polyacrylamide gel electrophoresis (SDS-PAGE) and transferred to a PVDF membrane (Millipore) using a semidry blotting apparatus (Bio-Rad Laboratories, Hercules, CA, USA). Membranes were blocked with $5 \%(\mathrm{w} / \mathrm{v})$ non-fat milk in TBST (100 mM NaCl, $50 \mathrm{mM}$ Tris and 0.1\% Tween-20) at room temperature for $1 \mathrm{~h}$ and then they were incubated overnight with primary antibodies at $4^{\circ} \mathrm{C}$. After washing three times with TBST, the membranes were incubated with appropriate HRP-labelled secondary antibodies (KPL) for $2 \mathrm{~h}$. Finally, the signals of protein bands were detected with an ECL system (Merck) according to the manufacturer's instructions. $\beta$-actin was used as the endogenous loading control.

Migration and invasion assay. For the migration assays, the lower chamber was filled with $500 \mu 1$ of conditional medium from NIHT3 cells. The cells were digested with trypsin and $1 \times 10^{5}$ cells suspended with serum-free MEM were seeded onto the upper chamber. After $16 \mathrm{~h}$, the non-migrating cells inside the filters were removed with a cotton swab and the migrating cells on the other side of the filter were fixed with methanol and stained with $0.1 \%$ crystal violet. The number of cells was counted in at least 10 random fields under a light microscope and data were analysed statistically.

For the invasion assays, a Transwell system $(8-\mu \mathrm{m}$ pore size; BD Biosciences, Bedford, MA, USA) precoated with $2 \mathrm{mg} / \mathrm{ml}$ of basement membrane Matrigel (BD Biosciences) was used. The cells were treated similar to the procedure used for the migration assay described above. After $24 \mathrm{~h}$, the filters were fixed and stained similar to the procedure used for the migration assay. Experiments were performed in triplicate.

Transient transfection assays. For the RNA interference assay, Lipofectamine 2000 (Invitrogen) was used according to the manufacturer's instructions. The cells were prepared at a density of $2 \times 10^{6}$ cells/flask and washed twice with PBS; then, a transfection mixture of NF- $\kappa$ B p65 siRNA was added 


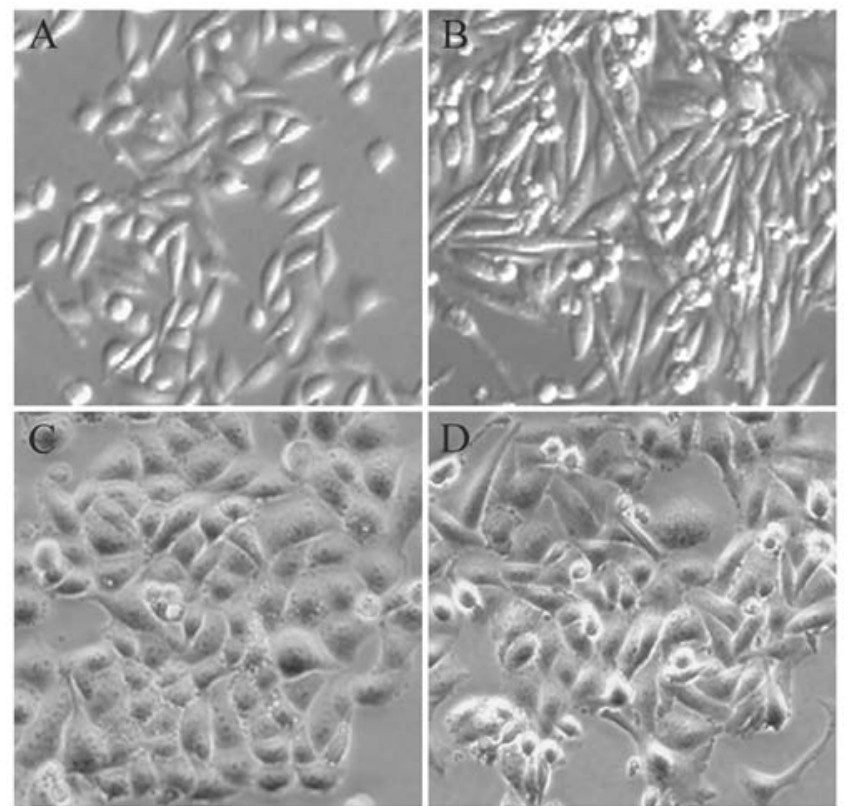

Figure 1. Morphology changes of cells undergoing low-dose radiation treatment. (A and C) The parental cell lines typically had a cobblestone-like shape. (B and D) The cells undergoing low-dose radiation treatment showed a spindle-like, elongated phenotype. (A and B) The human cervical cell line Siha. (C and D) The human cervical cell line C33A.

to the flasks. After a 4 -h incubation at $37^{\circ} \mathrm{C}$ in a $5 \% \mathrm{CO}_{2}$ in a humidified incubator, the transfection medium was discarded and replaced with complete medium.

Statistical analysis. Statistical analysis was performed using Student's t-test. Results are presented as the mean \pm standard deviation (SD). $\mathrm{P}<0.05$ was considered statistically significant and the experiments were repeated at least three times.

\section{Results}

The effects of low-dose radiotherapy on cellular morphology. When the treatment of parental cervical cancer cells with low-dose X-ray radiation reached the total of 20-Gy, the morphology of cells changed from a polarised epithelial cell type to a mesenchymal cell type. As shown in Fig. 1, the parental cells exhibited a typical cobblestone-like shape and tight cell junctions, which are traits of the epithelial phenotype. Additionally, the FIR cells treated with low-dose X-ray radiation demonstrated a spindle-like, elongated phenotype with loss of cell-to-cell contact, features that are characteristic of fibroblasts.

The effects of low-dose radiotherapy on the expression of mesenchymal and epithelial markers. Preview results showed that the low-dose radiation treatment induced the morphological changes of cells. To evaluate if this change was the result of epithelial-mesenchymal transition (EMT), we tested the expression of EMT markers in FIR cells and their parental cells. Real-time qPCR results showed that expression of the epithelial markers E-cadherin and CK-18 were remarkably downregulated $(\mathrm{P}<0.01)$ and the mesenchymal markers $\mathrm{N}$-cadherin and vimentin were upregulated in FIR cells. Additionally, the expression of N-cadherin in Siha FIR cells was highly significantly upregulated $(\mathrm{P}<0.01$, $\mathrm{P}=0.00066$ ) and the expression of vimentin in the two FIR cell lines was significantly upregulated compared with that of their parental cells $(\mathrm{P}<0.05)$ (Fig. 2A). Next, we determined the protein expression levels of the two FIR cell lines.

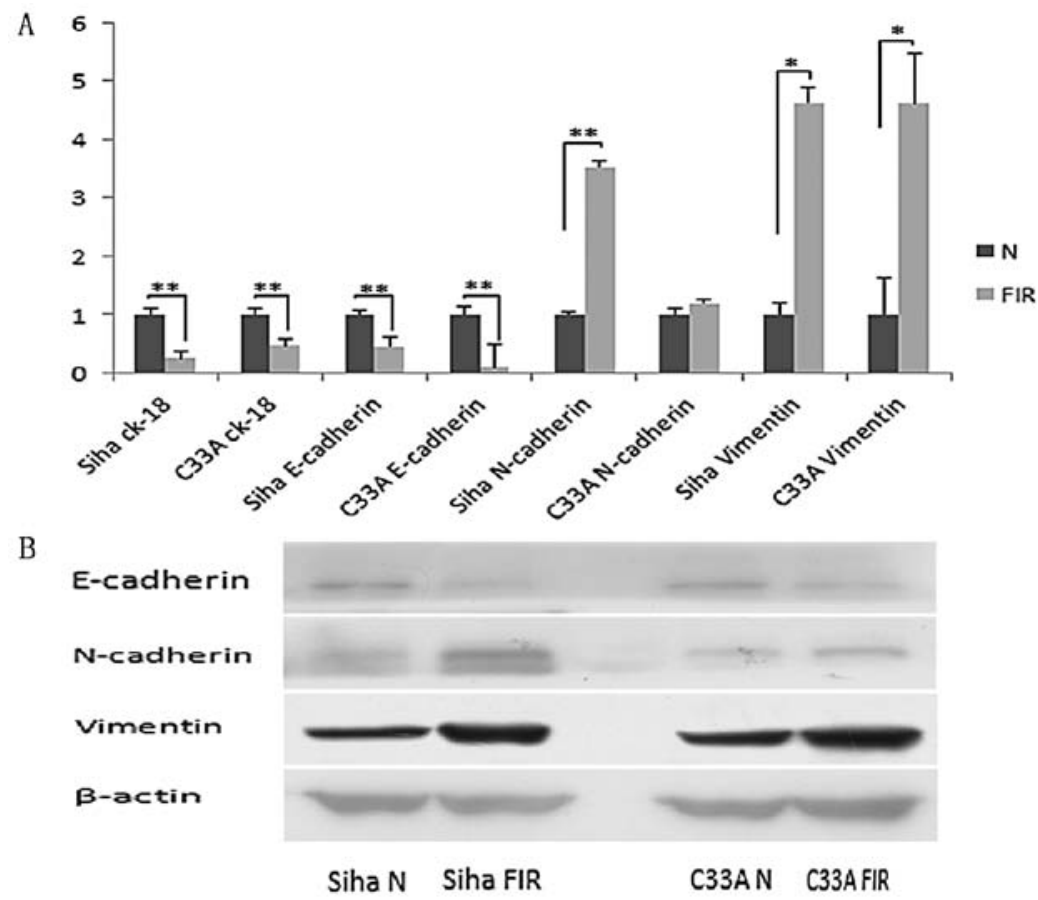

Figure 2. Expression changes of epithelial to mesenchymal transition (EMT) markers in parental cells and FIR cells induced by low-dose radiation treatment. (A) The mRNA levels were examined by real-time qPCR. Data are expressed as the mean \pm SD. (B) The protein levels were measured by western blot analysis. $\mathrm{N}$, the parental cell line; FIR, cells undergoing low-dose radiation treatment-induced EMT. $\beta$-actin was used as the endogenous loading control. 


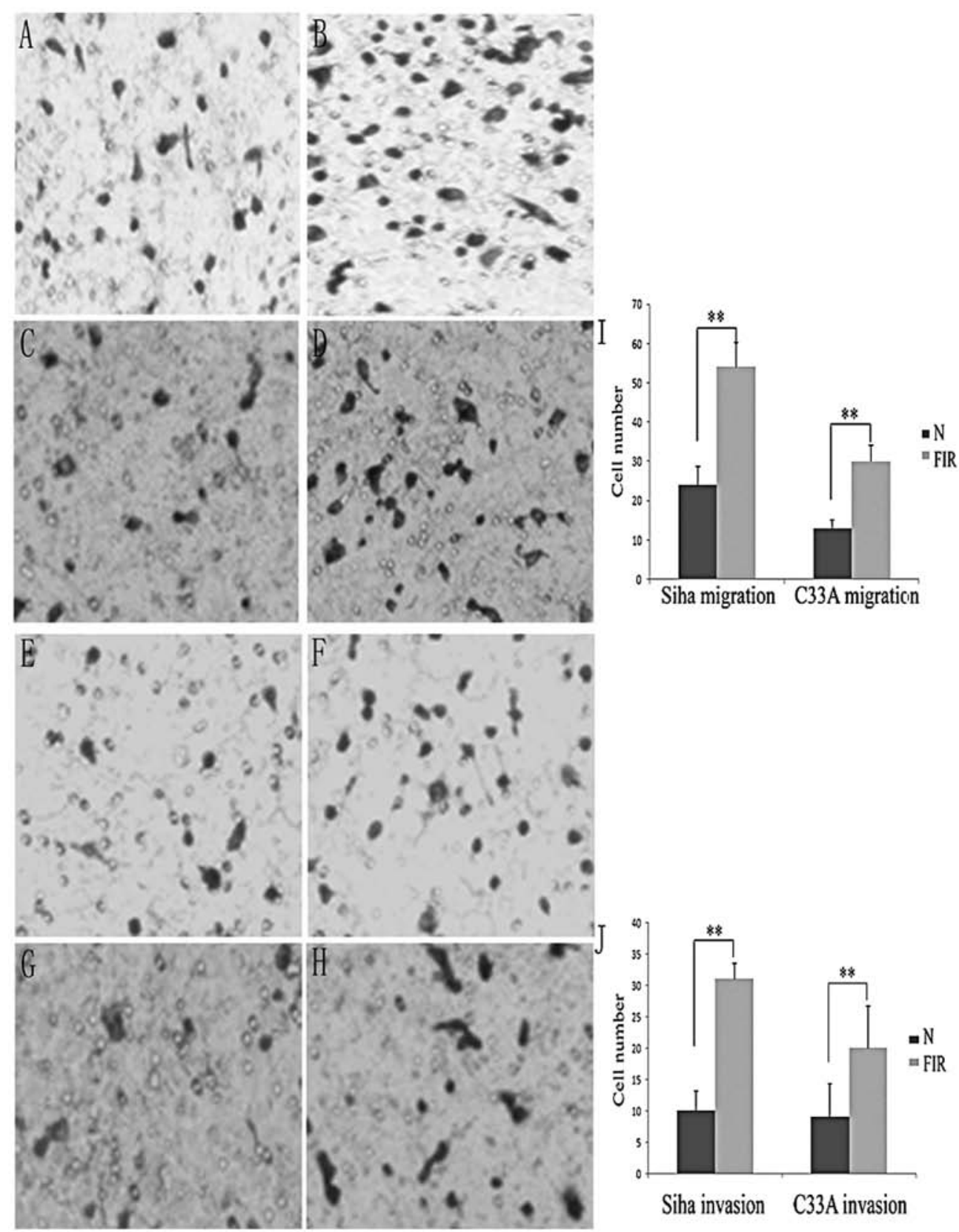

Figure 3. Low-dose radiation-induced EMT enhanced cell mobility. (A-D) The migration ability of the two FIR cell lines was significantly enhanced compared with their parental cells $(\mathrm{P}<0.01)$. (E-H) The 16-h invasion assay indicated that the two FIR cell lines acquired a stronger invasion ability than their parental cells $(\mathrm{P}<0.05)$. (A and E) Siha parental cells, (B and F) Siha FIR cells; (C and G) C33A parental cells; (D and H) C33A FIR cells.

Western blot analysis was consistent with these results. The expression of E-cadherin was decreased and N-cadherin and vimentin expression were increased compared with their parental cells (Fig. 2B). These results indicated that low-dose irradiation in cervical cancer cells induced EMT.

Low-dose ionising radiotherapy increases migration and invasion of cervical cancer cells. Cells that undergo EMT have demonstrated increased migration and invasion ability (1). To determine whether these FIR cells display such traits, we evaluated the migration and invasion ability of FIR cells using the two-chamber assay. As shown in Fig. 3, the migration ability of the two FIR cell lines $(\mathrm{P}<0.01)$ was significantly enhanced compared with that of their parental cells $(\mathrm{P}<0.05)$. The $16-\mathrm{h}$ invasion assay indicated that the two FIR cell lines acquired a stronger invasive ability than their parental cells (Fig. 3B).

Low-dose radiation-induced EMT is related to the $N F-\kappa B$ pathway. NF- $\mathrm{B}$ was reported to be involved in Snail generegulated EMT $(19,20)$. Additionally, our lab previously demonstrated that the p65 subunit of $\mathrm{NF}-\kappa \mathrm{B}$ plays a role in MTDH-induced EMT (21). To determine whether the p65 subunit of $\mathrm{NF}-\kappa \mathrm{B}$ was involved in the EMT process induced by low-dose radiation, we evaluated the expression of p65 and phosphorylated p65 by western blot analysis in parental and FIR cervical cancer cells. As shown in Fig. 4A, p65 expression was upregulated and phosphorylated p65 expression was downregulated in the two FIR cell lines. 
A

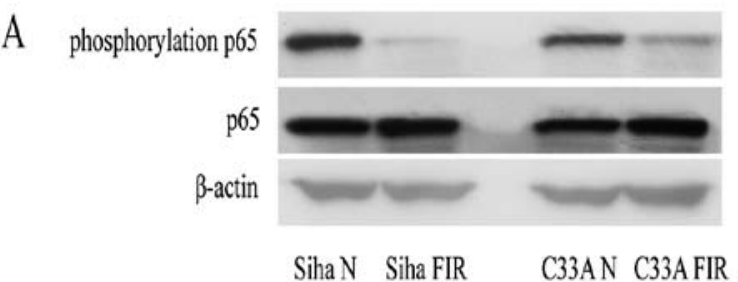

B

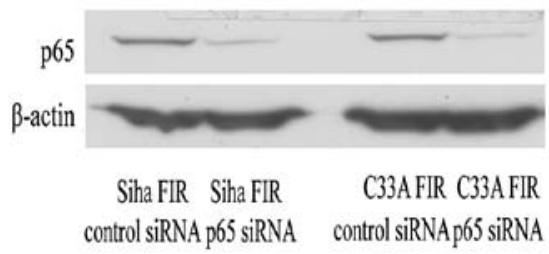

D
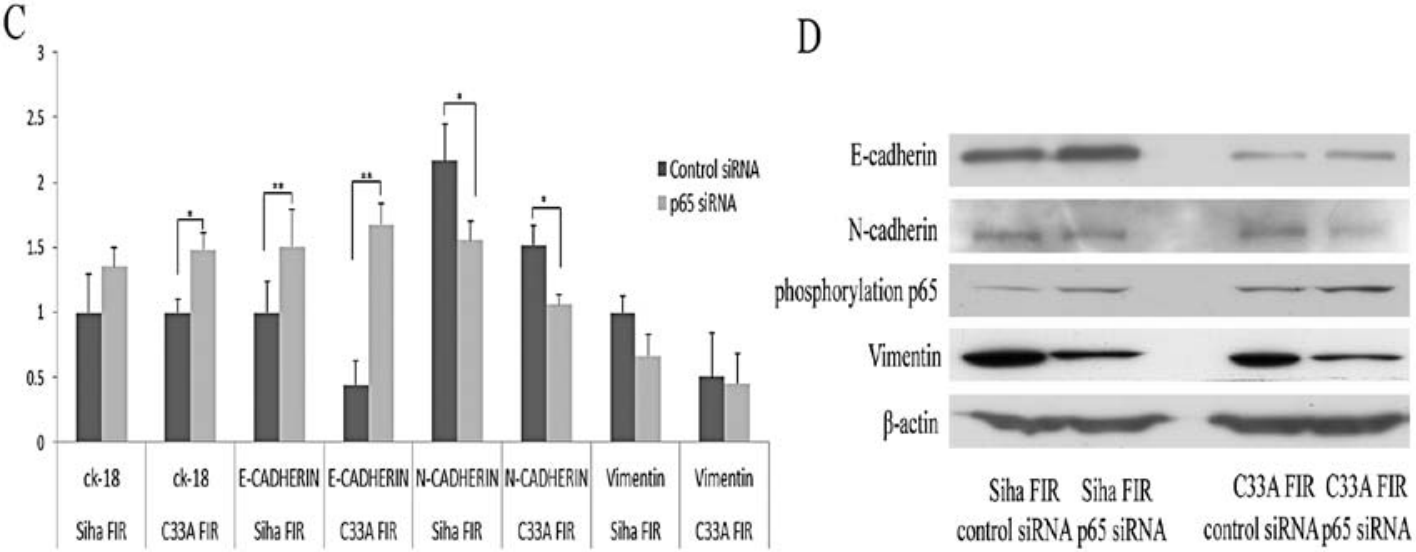

Figure 4. Effect of the NF- $\kappa$ B pathway in the low-dose radiation-induced EMT process. (A) Expression of the p65 subunit of NF- $\kappa \mathrm{B}$ in low-dose radiationinduced EMT. (B) Efficiency of transfection of p65. (C) Real-time qPCR results of EMT marker expression after transfection of p65 siRNA. The expression of E-cadherin in the two FIR cell lines was upregulated after transfecting p65 siRNA $\left({ }^{* *} \mathrm{P}<0.01\right)$ and the expression of N-cadherin was downregulated after transfecting p65 siRNA ( $\mathrm{P}<0.05)$. (D) Western blot analysis evaluated EMT marker expression after transfection of p65 siRNA. Expression of E-cadherin and phosphorylated p65 was upregulated after transfection of p65 siRNA and expression of the mesenchymal markers N-cadherin and vimentin were downregulated after transfection of p65 siRNA. N, the parental cell line; FIR, cells undergoing low-dose radiation treatment-induced EMT. $\beta$-actin was used as the endogenous loading control.

We then further tested the roles of $\mathrm{NF}-\kappa \mathrm{B}$ in low-dose radiation-induced EMT by transfecting NF- $\kappa \mathrm{B}$ p65 siRNA into FIR cells. The RNA interference efficiency of p65 was tested by western blot analysis $48 \mathrm{~h}$ after transfection (Fig. 4B). Real-time qPCR results showed that the miRNA expression of E-cadherin and CK-18 was upregulated and that of $\mathrm{N}$-cadherin and vimentin was downregulated after transfection with NF- $\kappa$ B p65 siRNA (Fig. 4C). After transfection with $\mathrm{NF}-\kappa \mathrm{B}$ p65 siRNA, E-cadherin expression was significantly upregulated $(\mathrm{P}<0.01)$ and the expression of $\mathrm{N}$-cadherin was downregulated $(\mathrm{P}<0.05)$. The results of western blot analysis of the EMT markers were consistent with the real-time qPCR results (Fig. 4D). These results suggested that p65 was involved in EMT induced by low-dose radiation.

\section{Discussion}

Radiotherapy is an effective curative method for cervical cancer, even when the disease is at an advanced stage. However, for some patients, it is easy to gain radioresistance (3) during the therapy process, causing therapy to become more difficult. Hence, radiation resistance remains a challenge for radiotherapy and understanding the molecular mechanism of radiation resistance is important to develop new therapeutic strategies against cervical cancer. Our results showed that low-dose radiation-induced EMT through $\mathrm{NF}-\kappa \mathrm{B}$ p65 signalling enhanced the migration and invasion of cervical cancer cells.

EMT is a morphogenesis process in multicellular organisms (22) that has been confirmed to cause the loss of cell adhesion molecules and to enhance cell migration and invasion abilities $(1,22,23)$, promoting tumour progression $(13,24)$. Cancer cells that undergo EMT gain malignant behaviour such as chemoresistance of cancer cells. In the present study, we showed that low-dose radiation treatment could induce the cervical cancer cell phenotype to switch from a cobblestonelike epithelial morphology to a spindle-shaped mesenchymal morphology. At the same time, expression of the epithelial markers E-cadherin and CK-18 was downregulated and that of the mesenchymal markers $\mathrm{N}$-cadherin and vimentin was upregulated in the FIR cells versus their parental cells. Our results further showed that the motility of FIR cells was enhanced compared with that of their parental cells.

$\mathrm{NF}-\kappa \mathrm{B}$ is a transcription factor that plays an important role in the regulation of cell apoptosis and tumourigenesis. Additionally, $\mathrm{NF}-\kappa \mathrm{B}$ is essential for EMT induction and maintenance $(19,25)$. p38 suppresses TAK1-NF- $\mathrm{B}$ signalling to maintain E-cadherin expression, impeding the induction of EMT in human primary mesothelial cells (26). NF- $\kappa \mathrm{B}$ signalling was involved in TNF- $\alpha$-induced expression of Twist 1 and overexpression of p65 induced upregulation of Twist1 expression together with EMT in mammary epithelial cells (27). Previous studies have shown that NF- $\kappa \mathrm{B}$ p65 is involved in EMT induced by MTDH in breast cancer cells (21). We further confirmed that $\mathrm{NF}-\kappa \mathrm{B}$ p 65 was involved in low-dose radiationinduced EMT in cervical cancer cells by RNA interference using NF- $\kappa$ B p65 siRNA. Our results showed that when NF- $\mathrm{B}$ p65 expression was silenced using siRNA, the EMT process was reversed. E-cadherin expression was upregulated, whereas expression of $\mathrm{N}$-cadherin was downregulated. 
These results suggested that p65 was associated with low-dose radiation-induced EMT of human cervical cancer.

In conclusion, continuous low-dose radiation enhanced the invasion potential of cervical cancer cells by inducing EMT. p65 played an important role in this process and silencing p65 could change this process. Targeting the reversion of EMT or inhibiting EMT and its regulators may provide a new therapeutic target for improving the effectiveness of radiotherapy of cervical cancer.

\section{Acknowledgements}

This study was supported by the National Natural Science Foundation of China (no. 81272857; 81072150), the Independent Innovation Foundation of Shandong University (grant nos. 2010TS084 and 2010TS085) and the Natural Science Foundation of Shandong (grant nos. ZR2009CQ038).

\section{References}

1. Lee MY and Shen MR: Epithelial-mesenchymal transition in cervical carcinoma. Am J Transl Res 4: 1-13, 2012.

2. Siegel R, Naishadham D and Jemal A: Cancer statistics, 2012. CA Cancer J Clin 62: 10-29, 2012.

3. Kitahara O, Katagiri T, Tsunoda T, Harima Y and Nakamura Y: Classification of sensitivity or resistance of cervical cancers to ionizing radiation according to expression profiles of 62 genes selected by cDNA microarray analysis. Neoplasia 4: 295-303, 2002.

4. Andarawewa KL, Erickson AC, Chou WS, et al: Ionizing radiation predisposes nonmalignant human mammary epithelial cells to undergo transforming growth factor beta induced epithelial to mesenchymal transition. Cancer Res 67: 8662-8670, 2007.

5. Qing Y, Yang XQ, Zhong ZY, et al: Microarray analysis of DNA damage repair gene expression profiles in cervical cancer cells radioresistant to $252 \mathrm{Cf}$ neutron and X-rays. BMC Cancer 10: 71, 2010.

6. Liu SS, Leung RC, Chan KY, et al: p73 expression is associated with the cellular radiosensitivity in cervical cancer after radiotherapy. Clin Cancer Res 10: 3309-3316, 2004.

7. Barcellos-Hoff MH: Radiation-induced transforming growth factor beta and subsequent extracellular matrix reorganization in murine mammary gland. Cancer Res 53: 3880-3886, 1993.

8. Ewan KB, Henshall-Powell RL, Ravani SA, et al: Transforming growth factor-betal mediates cellular response to DNA damage in situ. Cancer Res 62: 5627-5631, 2002.

9. Thiery JP, Acloque H, Huang RY and Nieto MA: Epithelialmesenchymal transitions in development and disease. Cell 139: 871-890, 2009.
10. Zeisberg M and Neilson EG: Biomarkers for epithelial-mesenchymal transitions. J Clin Invest 119: 1429-1437, 2009.

11. Thiery JP: Epithelial-mesenchymal transitions in tumour progression. Nat Rev Cancer 2: 442-454, 2002.

12. Moody SE, Perez D, Pan TC, et al: The transcriptional repressor Snail promotes mammary tumor recurrence. Cancer Cell 8: 197-209, 2005.

13. Lee MY, Chou CY, Tang MJ and Shen MR: Epithelialmesenchymal transition in cervical cancer: correlation with tumor progression, epidermal growth factor receptor overexpression and snail up-regulation. Clin Cancer Res 14: 4743-4750, 2008.

14. Hsu DS, Lan HY, Huang CH, et al: Regulation of excision repair cross-complementation group 1 by Snail contributes to cisplatin resistance in head and neck cancer. Clin Cancer Res 16: 4561-4571, 2010

15. Yang AD, Fan F, Camp ER, et al: Chronic oxaliplatin resistance induces epithelial-to-mesenchymal transition in colorectal cancer cell lines. Clin Cancer Res 12: 4147-4153, 2006.

16. Cheng GZ, Chan J, Wang Q, Zhang W, Sun CD and Wang LH: Twist transcriptionally up-regulates AKT2 in breast cancer cells leading to increased migration, invasion and resistance to paclitaxel. Cancer Res 67: 1979-1987, 2007.

17. Arumugam T, Ramachandran V, Fournier KF, et al: Epithelial to mesenchymal transition contributes to drug resistance in pancreatic cancer. Cancer Res 69: 5820-5828, 2009.

18. Li Z, Xia L, Lee LM, et al: Effector genes altered in MCF-7 human breast cancer cells after exposure to fractionated ionizing radiation. Radiat Res 155: 543-553, 2001.

19. Min C, Eddy SF, Sherr DH and Sonenshein GE: NF-kappaB and epithelial to mesenchymal transition of cancer. J Cell Biochem 104: 733-744, 2008.

20. Cho KB, Cho MK, Lee WY and Kang KW: Overexpression of c-myc induces epithelial mesenchymal transition in mammary epithelial cells. Cancer Lett 293: 230-239, 2010.

21. Li X, Kong X, Huo Q, et al: Metadherin enhances the invasiveness of breast cancer cells by inducing epithelial to mesenchymal transition. Cancer Sci 102: 1151-1157, 2011.

22. Thiery JP: Epithelial-mesenchymal transitions in development and pathologies. Curr Opin Cell Biol 15: 740-746, 2003.

23. Schmalhofer O, Brabletz S and Brabletz T: E-cadherin, betacatenin and ZEB1 in malignant progression of cancer. Cancer Metastasis Rev 28: 151-166, 2009.

24. Hsu YM, Chen YF, Chou CY, et al: $\mathrm{KCl}$ cotransporter-3 down-regulates E-cadherin/beta-catenin complex to promote epithelial-mesenchymal transition. Cancer Res 67: 11064-11073, 2007.

25. Huber MA, Azoitei N, Baumann B, et al: NF-kappaB is essential for epithelial-mesenchymal transition and metastasis in a model of breast cancer progression. J Clin Invest 114: 569-581, 2004.

26. Strippoli R, Benedicto I, Foronda M, et al: p38 maintains E-cadherin expression by modulating TAK1-NF-kappa B during epithelial-to-mesenchymal transition. J Cell Sci 123: 4321-4331, 2010.

27. Li CW, Xia W, Huo L, et al: Epithelial-mesenchymal transition induced by TNF-alpha requires NF-kappaB-mediated transcriptional upregulation of Twist1. Cancer Res 72: 1290-1300, 2012. 